\title{
Visual Motion Capturing for Kinematic Model Estimation of a Humanoid Robot
}

\author{
Andre Gaschler \\ gaschler@cs.tum.edu \\ Technische Universität München, Germany
}

\begin{abstract}
Controlling a tendon-driven robot like the humanoid ECCE is a difficult task, even more so when its kinematics and its pose are not known precisely. In this paper, we present a visual motion capture system to allow both real-time measurements of robot joint angles and model estimation of its kinematics.

Unlike other humanoid robots, ECCE (see Fig. 1A) is completely molded by hand and its joints are not equipped with angle sensors. This anthropomimetic robot design [5] demands for both (i) real-time measurement of joint angles and (ii) model estimation of its kinematics. The underlying principle of this work is that all kinematic model parameters can be derived from visual motion data. Joint angle data finally lay the foundation for physics-based simulation and control of this novel musculoskeletal robot.
\end{abstract}
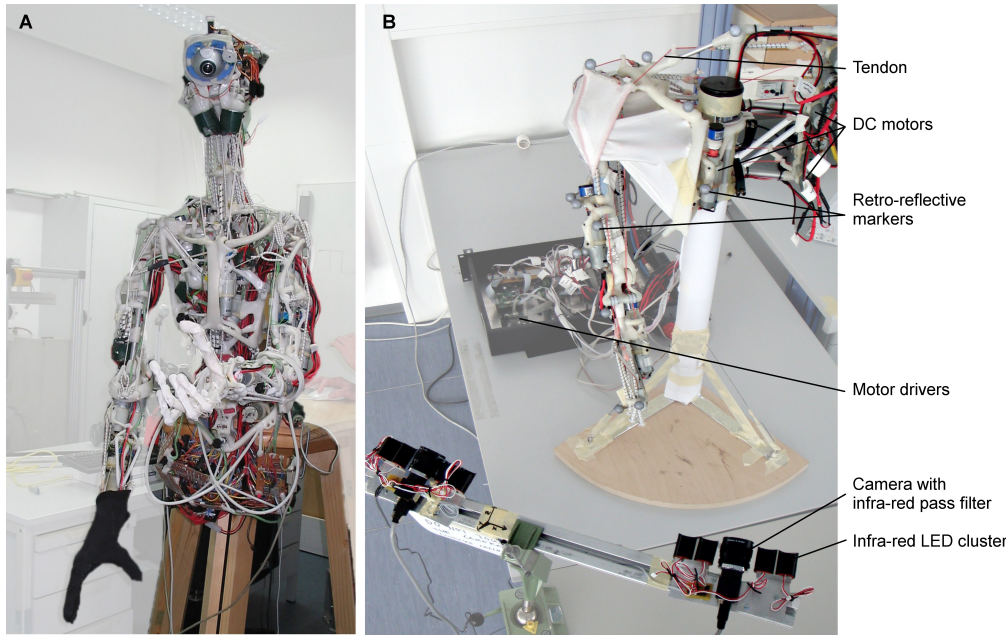

Fig. 1. A: Musculoskeletal humanoid robot ECCE B: Shoulder test rig with visual motion capture system, both robots developed within the ECCEROBOT project [6]

Recommended for submission to YRF2011 by supervisor Alois Knoll 


\section{Introduction}

As for almost all robot control tasks, modeling the kinematic structure and obtaining real-time joint angle data is of crucial importance. Controlling the muscle-based humanoid robot ECCE (Fig. 1A) is still an unresolved problem, but without knowledge of its precise kinematics, only few control approaches can be used at all [1]. However, the novel muscle-based humanoid ECCE is completely molded by hand in a rapid-prototyping process, its skeleton is hand-crafted using the thermoplastic polymorph [6] and its artificial muscles are made of tendondriven actuators. Therefore, we first need to estimate its kinematic parameters in order to allow approaches to robot simulation and control.

Beyond the need for precise kinematic parameters, real-time measurement of joint angles is also of crucial importance for robot controller design. However, the robot is equipped with ball-and-socket joints, in which direct angle sensors can hardly be incorporated. Creating a three-dimensional angle sensor for a spherical joint is a challenging task: For another tendon-driven robot Kotaro, Urata et al. developed a custom-made sphere joint angle sensor using a micro camera and image processing of markers in the joint socket [11].

Our requirements are slightly different, as we need a means for joint angle measurement that is inexpensive, commercially available and very precise, but not necessarily internal. We therefore decided to use external motion sensing, which can be dedicatedly installed and calibrated at all three robots of the EсCERовот project. For that, we first tested a Polhemus Liberty ${ }^{\mathrm{TM}}$ magnetic motion capture system. However, the magnetic sensors showed a jitter of up to $5 \mathrm{~mm}$ and 3 degrees during motor operation, rendering the magnetic tracking approach impractical for our setup. After further review of motion capture systems, we decided on a visual stereoscopic solution with passive retro-reflective marker balls and infra-red illumination, similar to [7]. This solution is available from commodity hardware, cost-effective and allows us to arrange the markers over the full length of the robot's limbs, effectively increasing the precision of orientation and joint angles in comparison to systems with fixed marker sizes. In the following, the setup of our motion capture system is briefly described.

\section{Visual Motion Capture System}

The overall setup of our motion capture system is shown in Fig. 1B. Each robot limb is equipped with 4 to 6 marker spheres with retro-reflective coating. A stereo camera setup of two PointGrey Flea 2 cameras with $6 \mathrm{~mm}$ Pentax optics and a baseline of $477 \mathrm{~mm}$ is installed roughly $1 \mathrm{~m}$ from the robot. Each camera is enclosed by four $\lambda=880 \mathrm{~nm}$ LED clusters and equipped with $\lambda_{\text {thresh }}=750 \mathrm{~nm}$ infra-red pass filters.

Marker thresholding, connected component search and 2D coordinate extraction are efficiently implemented at sub-pixel accuracy similar to the standard methods [7]. After that, the 3D coordinates of the marker balls are obtained by optimal 3D triangulation. All these image processing steps are described at length in [4]. 


\subsection{Efficient Rigid Body Detection}

Once the 3D marker positions are available, the combined matching and orientation problem of the known rigid marker targets needs to be solved in order to recover the poses of the robot's limbs. Mathematically, the rigid body detection is the problem of aligning a selection $\Pi_{M}$ of $m$ from $k$ known marker points $M$ with a selection $\Pi_{P}$ of $m$ from $n$ measured points $P$ under a rigid transformation $R T . \Pi_{M}$ and $\Pi_{P}$ are binary matrices that select and permute $3 \mathrm{D}$ points of $M$ and $P$, respectively. Our rigid body detection step finds a compromise between the number of matching points $m$ and the residual geometric error of the alignment:

$$
\underset{\Pi_{M}, \Pi_{P}, R T}{\arg \min }\left\|\Pi_{P} P-R T \Pi_{M} M\right\|_{2} \frac{1.5^{k-m}}{m} \text { s.t. } m \geq 3
$$

Here, the constant 1.5 is a design parameter to penalize low numbers of matching points. Even though this problem is similar to the largest clique search and can be theoretically infeasible for even small numbers of points, we can dramatically shrink the search space by applying an upper threshold $t$ that rejects all matchings over a certain geometric distance, in our case $t=5 \mathrm{~mm}$. As an initial step, a priority queue of 2-matchings is built, which can be ordered by geometric distance in $O\left(n^{2}\right)$ [7]. From that, we select only a certain quantile, in our case the best 50 matchings - note that this is the only heuristic we apply in our algorithm. On this set, the actual search is conducted in a RANSAC-like fashion [2], recursively adding candidate points. In every recursive step, the residual geometric distance is checked against the threshold $t$, leaving very few evaluations for real-world problems [4]. For $m \geq 3$, transformations $R T$ are recovered by Umeyama's method [10]. Finally, the poses $R T$ of the limbs of the robot are output.

We believe that our approach is particularly efficient thanks to the heavily pruned search tree, compared to the extensive search in [8] or a maximum-clique search [7]. Furthermore, it is able to handle very low numbers of inliers in contrast to rigid point set registration approaches based on interative closest point [9] or eigenstructure decomposition [12].

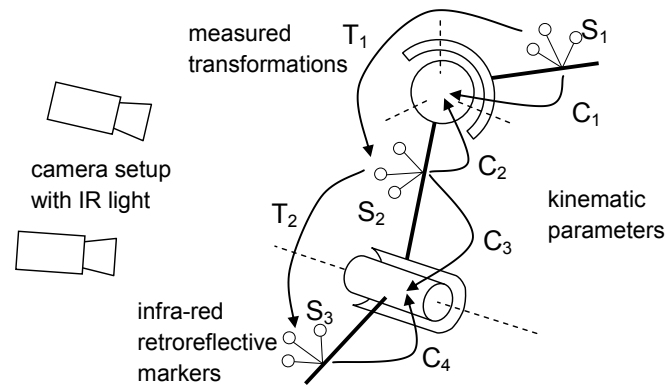

Fig. 2. Kinematic parameter estimation using visual motion capturing 


\section{Kinematic Model Estimation and Joint Angle Calculation}

Now that the pose of the robot is available from the motion capture system, we are to calibrate its kinematic model and then calculate the joint angles.

\subsection{Ball Joint Model Estimation}

First, we consider the calibration of ball-and-socket joints based on the method described in [3]. As shown in Fig. 2, a ball joint can be parameterized by the position of the center of rotation with respect to the two frames of reference given by the attached marker targets. Let $c_{1}$ and $c_{2}$ be the rotational center in the reference frames $S_{1}$ and $S_{2}$, respectively. Measuring several joint poses $T_{i}$, we can assume $c_{1} \approx T_{i} c_{2}$ for all $i$. Separating the rotational and translational parts of $T_{i}$ such that $T_{i}=\left[R_{i} t_{i}\right]$, we obtain a linear least squares problem:

$$
\underset{c_{1}, c_{2}}{\arg \min } \underbrace{\left[\begin{array}{cc}
I & -R_{1} \\
I & -R_{2} \\
\vdots
\end{array}\right]}_{M}\left[\begin{array}{c}
c_{1} \\
c_{2}
\end{array}\right]-\left[\begin{array}{c}
t_{1} \\
t_{2} \\
\vdots
\end{array}\right]
$$

This problem is easily solved by standard numerical libraries and we obtain the kinematic parameters $c_{1}$ and $c_{2}$.

\subsection{Hinge Joint Model Estimation}

As hinge joints are essentially a special case of ball-and-socket joints, we can again apply Eq. 2. However, the minimization then yields a random point on the rotational axis of the hinge joint, possibly far away from the physical setup. Gamage et al. [3] resolve the rotational axis ambiguity by replacing the measurement matrix $M$ by its closest rank-5 approximation $M_{5}$, which leads to a well-defined position for the center of rotation $c$. The null space of $M_{5}$ yields the axis of rotation $c_{z}$ in both reference frames, which we define as the $z$-axis of the rigid transformations to the axis coordinate frames. With the further choice $c_{y}=c \times c_{z}$ and $c_{x}=c_{z} \times c_{y}$ and normalization to unit vectors, we finally obtain a unique parameterization of the hinge joint coordinate frame $C=\left[\begin{array}{lll}c_{x} & c_{y} & c_{z}\end{array}\right]$, for $C_{3}$ and $C_{4}$, respectively. As described in [4], we further perform a non-linear minimization on our kinematic model in order to minimize the actual marker ball residual errors.

Finally, we have obtained a unique parameterization for both ball joints and hinge joints. This allows us to model the kinematics of the robot ECCE. For the shoulder test rig in Fig. 1B, we measured 22 distinct joint poses from several viewpoints and could calibrate the robot kinematics up to a residual error of $1.29 \mathrm{~mm}$ for the position of the center of rotation and $0.83 \mathrm{~mm}$ for the axis of rotation. Note that this error is far better than in earlier manual measurements, when we could estimate the robot's kinematics at an error of $\approx 10 \mathrm{~mm}$. 


\begin{tabular}{|c|c|}
\hline A. Accuracy & of $3 \mathrm{D}$ position \\
\hline Marker Targe & Position $[\mathrm{mm}$ \\
\hline$S_{1}$ Torso & 0.8082 \\
\hline$S_{2}$ Upper Arn & 0.8214 \\
\hline$S_{3}$ Lower Ar & 1.2083 \\
\hline
\end{tabular}

\begin{tabular}{|llll|}
\hline \multicolumn{4}{|l|}{ B. Precision of joint angles } \\
\hline \multicolumn{4}{|c|}{ Translation Rotation } \\
Transformation $[\mathrm{mm}$ ] & [degrees] & [degrees] \\
\hline$T_{1}$ Shoulder & 0.4833 & 0.2382 & 0.2060 \\
$T_{2}$ Elbow & 0.5638 & 0.2304 & 0.0546 \\
\hline
\end{tabular}

Table 1. Error Evaluation Results

\subsection{Joint Angle Calculation}

With the kinematic parameters at hand, we finally calculate joint angles given the transformations $T$ from Section 2. For ball joints, the rotation can be recovered from the measured pose $T$ by solving the orthogonal Procrustes problem as described in [10]. For hinge joints, the angle calculation reduces to a 2 -dimensional problem in the plane perpendicular to the rotational axis. The rotation angle $\alpha$ can be obtained by employing the two-valued arctangent function, details are given in [4]. Our final motion capture system delivers real-time joint angle data at a 20-30 ms delay on a dual core $2.4 \mathrm{GHz}$ system.

\subsection{Error Evaluation}

In order to verify the accuracy of our motion capture system, we evaluated both the accuracy of $3 \mathrm{D}$ positions compared to known motions over a fixed distance, as well as the precision of all data while the changing camera angle.

First, the robot setup was moved over a known distance of $400 \mathrm{~mm}$, while the joint angles were unchanged. This measurement was repeated several times and under several angles, the root mean square error of measured distances compared to the known distance is shown in Table 1A.

Second, we measured the precision of motion capture (see Table 1B) data while moving the camera to widely different angles over a sequence of 2000 frames. It is our strong belief that most sources of errors - except overall scalingwill show up when changing the viewpoint. From these results, we draw the conclusion that our system delivers joint angles at an error well below 1 degree.

\section{Conclusion}

In this work, we have developed a versatile motion capture system that serves two purposes: First, we can estimate the kinematic model of the musculoskeletal humanoid ECCE. Second, we can deliver real-time data of its pose and its joint angles, which opens up several areas of application. Both static and dynamic data may be captured and put to use for our future work in robot simulation and control.

\subsection{Future Work}

One of the central objectives of the ECCEROBOт project is to employ physicsbased robot simulation both off-line for controller development as well as online as an internal model for robot control $[6,5]$. Our motion capture system 
is therefore of great use for simulation parameter estimation and creation of a simulation model. Evolution strategies are currently applied in order to optimize the physics-based simulation model based on our joint angle measurements [13].

\section{Acknowledgments}

The author would like to thank Konstantinos Dalamagkidis and Alois Knoll (Robotics and Embedded Systems, Technische Universität München) for their valuable advice, as well as Steffen Wittmeier for his help with the robot platform.

\section{References}

1. Cheah, C., Liu, C., Slotine, J.: Adaptive tracking control for robots with unknown kinematic and dynamic properties. Intl J of Robotics Research 25(3), 283 (2006)

2. Fischler, M., Bolles, R.: Random sample consensus: A paradigm for model fitting with applications to image analysis and automated cartography. Communications of the ACM 24(6), 381-395 (1981)

3. Gamage, S., Lasenby, J.: New least squares solutions for estimating the average centre of rotation and the axis of rotation. J of Biomechanics 35(1), 87 (2002)

4. Gaschler, A.: Real-Time Marker-Based Motion Tracking: Application to Kinematic Model Estimation of a Humanoid Robot. Master's thesis, Technische Universität München, Germany (2011)

5. Jäntsch, M., Wittmeier, S., Knoll, A.: Distributed Control for an Anthropomimetic Robot. In: Intelligent Robots and Systems, Intl Conf on. pp. 5466-5471 (2010)

6. Marques, H., Jäntsch, M., Wittmeier, S., Holland, O., Alessandro, C., Diamond, A., Lungarella, M., Knight, R.: Ecce1: The first of a series of anthropomimetic musculoskeletal upper torsos. In: Humanoid Robots. pp. 391-396 (2010)

7. Pintaric, T., Kaufmann, H.: Affordable infrared-optical pose-tracking for virtual and augmented reality. In: Proc of Trends and Issues in Tracking for Virtual Environments Workshop. pp. 44-51 (2007)

8. Steinicke, F., Jansen, C., Hinrichs, K., Vahrenhold, J., Schwald, B.: Generating Optimized Marker-based Rigid Bodies for Optical Tracking Systems. In: Intl Conf on Computer Vision Theory and Applications (2007)

9. Trucco, E., Fusiello, A., Roberto, V.: Robust motion and correspondence of noisy 3-D point sets with missing data. Pattern recognition letters 20(9), 889-898 (1999)

10. Umeyama, S.: Least-squares estimation of transformation parameters between two point patterns. Pattern Analysis and Machine Intelligence pp. 376-380 (1991)

11. Urata, J., Nakanishi, Y., Miyadera, A., Mizuuchi, I., Yoshikai, T., Inaba, M.: A three-dimensional angle sensor for a spherical joint using a micro camera. In: Proc Intl Conf on Robotics and Automation. pp. 4428-4430 (2006)

12. Wang, X., Cheng, Y.Q., Collins, R.T., Hanson, A.R.: Determining correspondences and rigid motion of $3-\mathrm{d}$ point sets with missing data. In: Computer Vision and Pattern Recognition. pp. 252-257 (1996)

13. Wittmeier, S., Gaschler, A., Jäntsch, M., Dalamagkidis, K., Knoll, A.: Calibration of a physics-based model of an anthropomimetic robot using evolution strategies. In: Intelligent Robots and Systems, Intl Conf on (2011), submitted 